\title{
Comparison of Spin-Echo and Gradient-Echo T1-Weighted and Spin-Echo T2-Weighted Images at 3T in Evaluating Term-Neonatal Myelination
}

\author{
A.E. Tyan, A.M. McKinney, T.J. Hanson, and C.L. Truwit
}

\begin{abstract}
SUMMARY: A prior clinical report of 3T MR imaging in subsequently healthy very premature neonates imaged at term-equivalent age found that both gradient recalled-echo-TIWI and spin-echo-T2WI showed higher rates of myelinated structures, compared with spinecho-TIWI. The current study set out to assess those rates on the same sequences at $3 \mathrm{~T}$ in term neonates and thus consisted of 16 term neonates with normal-appearing MR imaging findings who subsequently had normal findings at clinical follow-up. Two neuroradiologists independently assessed 19 structures in those infants on all 3 sequences. Gradient recalled-echo-TIWI showed a slightly higher rate of myelination (57.2\%-72.4\% of all structures) and interobserver agreement $(\kappa=0.546, P<.0001)$ than spin-echo-T2WI $(58.2 \%-64.8 \% ; \kappa=$ $0.468, P<.0001)$, while spin-echo-TIWI had the lowest myelination rate and agreement $(25.0 \%-48.4 \% ; \kappa=0.384, P<.0001)$. Both observers noted that the following structures were myelinated in $88 \%-100 \%$ of patients on gradient recalled-echo-TIWI: the brachium of the inferior colliculus, decussation of the superior cerebellar peduncle, habenular commissure, medial lemniscus, pyramidal decussation, posterior limb of the internal capsule, and superior cerebellar peduncle; on spin-echo-T2WI, there was myelination in $88 \%-100 \%$ of the following structures: the brachium of the inferior colliculus, decussation of the superior cerebellar peduncle, inferior cerebellar peduncle, medial lemniscus, and posterior limb of the internal capsule. In conclusion, this study confirmed that similar to the findings in termequivalent-age premature infants, myelination changes in term neonates may be best assessed on both gradient recalled-echo-TIWI and spin-echo-T2WI at 3T, and not on spin-echo-TIWI.
\end{abstract}

ABBREVIATIONS: BIC = brachium of the inferior colliculus; CST = corticospinal tracts; DSCP $=$ decussation of the superior cerebellar peduncle; GRE $=$ gradient recalled-echo; ICP = inferior cerebellar peduncle; PLIC = posterior limb of the internal capsule; SCP = superior cerebellar peduncle; SE = spin-echo

M $\mathrm{R}$ imaging is often used in term neonates to assess myelination and to evaluate hypoxic-ischemic injury, developmental malformations, metabolic diseases, intracranial infections, and vascular injuries such as stroke or venous sinus thrombosis. ${ }^{1}$ While the process of myelination in term neonates has been well-documented histologically, there is a need to confirm such myelination by MR imaging; thus, previous studies at lower field strengths $(\leq 1.5 \mathrm{~T})$ have described normal myelination patterns in both term and preterm neonates on conventional spin-echo (SE)-T1WI and SE-T2WI sequences. $^{2,3}$ However, these patterns could differ at higher field strengths, where there are expectable gains in signal-to-noise and contrast-to-noise ratios, and the gray-white matter differentiation on

Received April 28, 2014; accepted after revision July 8.

From the Department of Radiology, University of Minnesota-Fairview and Hennepin County Medical Centers, Minneapolis, Minnesota.

Paper previously presented as an oral presentation at: Annual Meeting of the American Society of Neuroradiology and the Foundation of the ASNR Symposium, April 21-16, 2012; New York, New York.

Please address correspondence to Alexander M. McKinney, MD, Department of Radiology, University of Minnesota-Fairview and Hennepin County Medical Centers, Minneapolis, MN 55455; e-mail: mckinrad@umn.edu

http://dx.doi.org/10.3174/ajnr.A4099
SE-T1WI may worsen. ${ }^{4,5}$ Thus, there are few data comparing termneonatal myelination among gradient recalled-echo (GRE)-T1WI, SE-T1WI, and SE-T2WI at 3T in subsequently healthy term neonates. However, a recent preliminary study of subsequently developmentally healthy very preterm neonates evaluated by $3 \mathrm{~T}$ MR imaging at term-equivalent age showed that both GRE-T1WI and SE-T2WI had much higher rates of myelinated structures than SE-T1WI, with certain structures being visible on both GRE-T1WI and SE-T2WI in all infants: namely, the decussation of the superior cerebellar peduncle (DSCP), inferior cerebellar peduncle (ICP), and lateral lemniscus. ${ }^{6}$ That study also found that there was myelination in $90 \%-100 \%$ of these term-equivalent patients in the medial lemniscus, pyramidal decussation, posterior limb of the internal capsule (PLIC), and superior cerebellar peduncle (SCP) based solely on GRE-T1WI, and within the spinal tract of $\mathrm{V}$ (ie, the spinal trigeminal tract) based solely on SE-T2WI.

The purpose of this study was to assess the rates of myelination as determined by using these 3 sequences in a group of subsequently healthy term neonates who were initially evaluated for suspected hypoxic-ischemic injury and had normal MR imaging findings according to 2 pediatric neuroradiologists. Our goal was 

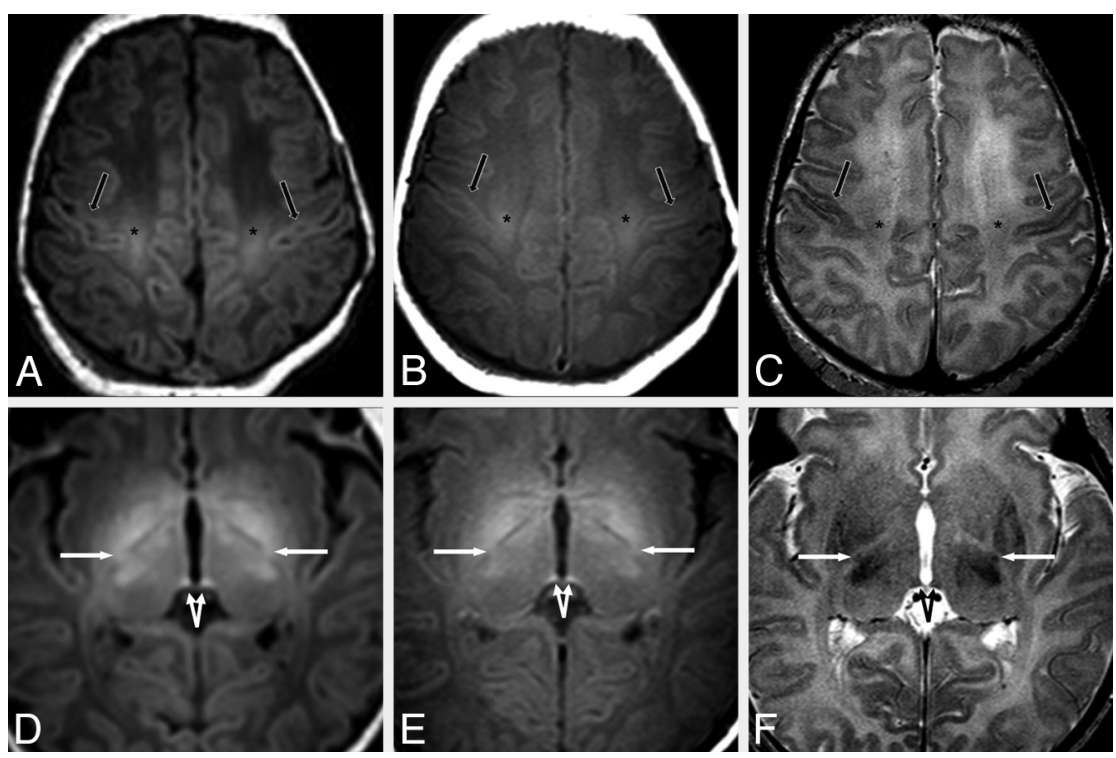

FIG 1. A 3-day-old male neonate with respiratory depression. A-C, MR images at the level of the centrum semiovale demonstrate mildly bright signal of the perirolandic cortex (black arrows) on GRE-TIWI (A) and SE-TIWI (B). The perirolandic cortex appears darker on SE-T2WI (C) relative to the remainder of the frontal and parietal cortices in most, but not all, infants in this study. The CST (asterisk) are also quite bright on both TIWI sequences and are mildly dark on SE-T2WI. D-F, On images at a lower level through the basal ganglia, the PLIC (arrows) and the habenular commissure (split arrows) appear visibly myelinated (ie, bright) on GRE-TIWI (D), SE-TIWI (E), and on SE-T2WI $(F)$, though the PLIC appears much smaller on SE-T2WI. Both the habenular commissure and the PLIC appear myelinated on GRE-TIWI in all term neonates in this study.

to determine whether the degree of myelination on those 3 sequences at $3 \mathrm{~T}$ matched that of prior studies at lower field strengths and matched the findings of a prior 3T study of preterm neonates imaged at term-equivalent age. ${ }^{2,3,6}$

\section{MATERIALS AND METHODS}

Institutional review board approval was obtained. PACS data base and clinical record reviews identified all term neonates younger than 21 days of age with a 3 T MR imaging to evaluate for hypoxicischemic injury between July 2006 and July $2012(n=49)$, with the intent of including only subsequently developmentally healthy children. Of the 49 term neonates, we excluded 33 on the basis of the following: cerebral structural pathology, hypoxicischemic injury, or parenchymal hematoma on MR imaging $(n=$ $15) ; 1$ of the 3 sequences missing $(n=9)$; neurologic deficit on clinical follow-up $(n=3)$; parenchymal calcification $(n=1)$; meningitis $(n=1)$; no clinical follow-up at older than 6 months of age $(n=3)$; or motion precluding evaluation $(n=1)$. Thus, we included 16 term neonates with normal initial MR imaging findings who were considered subsequently developmentally healthy on the basis of clinical evaluations at older than 6 months of age. At 3T, 3-mm axial GRE-T1WI, SE-T1WI, and SE-T2WI sequences are standard in our noncontrast MR imaging evaluation for suspected hypoxic-ischemic injury. Of the 16 remaining patients included for analysis, 10 were male and 6 were female. The recorded 5-minute Apgar scores of those patients ranged from 3 to 9 , with a median score of 7 .

\section{MR Imaging Technique}

All patients were scanned on a single 3T magnet (Intera; Philips Healthcare, Best, the Netherlands), with patients sedated by a pe- diatric intensivist. The parameters were identical to those of a prior study of very preterm neonates at 3T, which, for GRET1WI, were the following: a volumetric acquisition of $9.8 / 4.6 \mathrm{~ms} / 8^{\circ} / 15-20 \mathrm{~cm} / 1$ (TR/TE/flip angle/FOV/NEX), a $169 \times$ $169-240 \times 240$ matrix, 1 -mm-thickness reconstructed axially at $3 \mathrm{~mm}(0-\mathrm{mm}$ gap), and an acquisition time of 5 minutes. For SE-T1WI, the parameters were the following: 353-734/10 ms/14-20 cm/1 (TR/TE/FOV/NEX), a $168 \times 132$ $265 \times 205$ matrix, an axial thickness of 3 $\mathrm{mm}$ (0.3-mm gap), and an acquisition time of 5 minutes. For SE-T2WI, the parameters were the following: a turbo factor of fourteen, 3000/80-122 ms/14-20 cm/2 (TR/TE/FOV/NEX), a $268 \times 190-$ $400 \times 312$ matrix, an axial thickness of 3 $\mathrm{mm}$ (0.3-mm gap), and an acquisition time of approximately 2.5 minutes. $^{6}$

\section{Image Interpretation}

Two staff neuroradiologists (A.M.M., C.L.T., each with at least 10 years' experience in interpreting neonatal MR imaging) independently assessed each of the 19 structures, evaluating each sequence separately. The structures assessed were based on previous studies and included the following: brachium of the inferior colliculus (BIC), callosal splenium, cranial nerve $\mathrm{V}$ fascicle, corticospinal tracts (CST) within the brain stem, CST within the centrum semiovale, DSCP, habenular commissure, ICP, lateral geniculate nucleus, lateral lemniscus, medial lemniscus, medial longitudinal fasciculus, optic nerves, optic tracts, pyramidal decussation (being in close proximity to the decussation of the medial longitudinal fasciculus), PLIC, perirolandic cortex, SCP, and the spinal tract of V. ${ }^{2,3,6}$ The 2 staff reviewers consulted a dedicated imaging atlas to confirm the location of smaller brain stem structures before review. ${ }^{7}$ Each of the 19 structures was recorded as being "definitely myelinated" ( + , being relatively hyperintense on GRE-/SE-T1WI or hypointense on SE-T2WI compared with surrounding structures or periventricular white matter), "nonmyelinated" (-), or "equivocal" $( \pm)$, identical to the method of the prior study of very preterm neonates at $3 \mathrm{~T}^{6}{ }^{6}$ Representative examples of myelination assessment in 16 of the 19 structures are demonstrated in Figs 1-4, with the exception of the callosal splenium and the medial longitudinal fasciculus because those 2 structures had very low rates of myelination in this study (see the "Results" section).

Regarding statistical analysis, the overall percentage of myelination rates of all structures and the interobserver reliability $(\kappa)$ for each structure were calculated on the basis of each sequence. Later, after reviewing the medical records to determine which patients had undergone therapeutic head cooling within the first 72 hours, a Mann-Whitney $U$ test was implemented to determine whether there were overall any significant differences between 

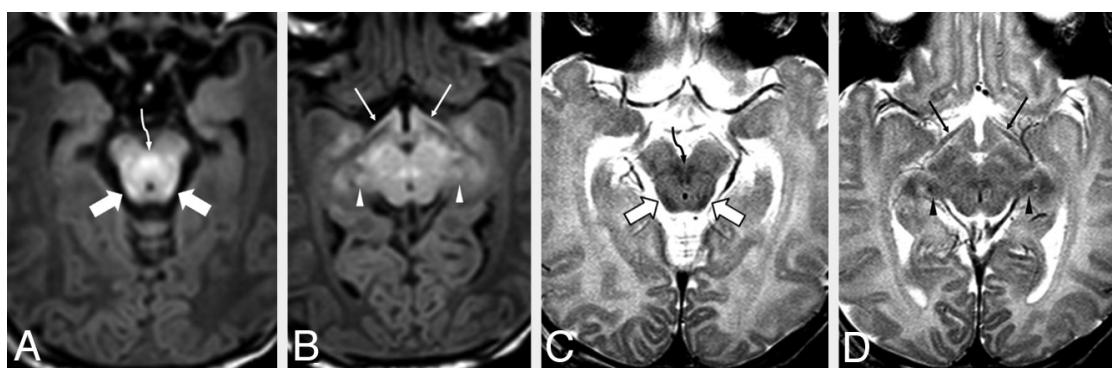

FIG 2. A 3-day-old male neonate with respiratory depression. MR images demonstrate clearly visible bright signal on GRE-TIWI ( $A$ and $B$ ) and clearly visible dark signal on SE-T2WI (C and D), indicative of normal early myelination within the BIC (wide arrows) and the DSCP (curved arrows). The optic tracts (thin arrows) are visibly myelinated on GRE-TTWI but are questionably myelinated on SE-T2WI. The BIC and the DSCP were both clearly myelinated in at least $\geq 88 \%$ of patients on both GRE-TIWI and SE-T2WI, while the SCP was myelinated in $\geq 88 \%$ of patients on GRE-TIWI only. Note the apparently myelinated lateral geniculate nuclei (arrowheads).
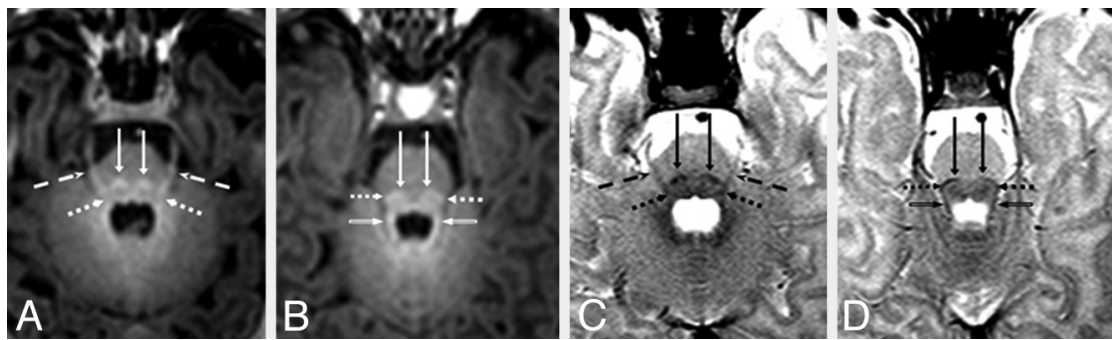

FIG 3. A 6-day-old male neonate with respiratory depression. Axial MR images illustrate bright myelination of the medial lemnisci (long arrows), lateral lemnisci (dotted arrows), cranial nerve $\mathrm{V}$ fascicle (dashed arrows), and SCP (beveled arrows) on GRE-TIWI ( $A$ and $B$ ), which were not clearly visible on SE-TIWI (not shown), but all appeared dark (myelinated) on SE-T2WI (C and D). The medial lemnisci were visibly myelinated in $\geq 94 \%$ of neonates on both GRE-TIWI and SE-T2WI. In most patients, the lateral lemnisci and cranial nerve $\mathrm{V}$ fascicle were best visualized as being myelinated on GRE-TIWI.
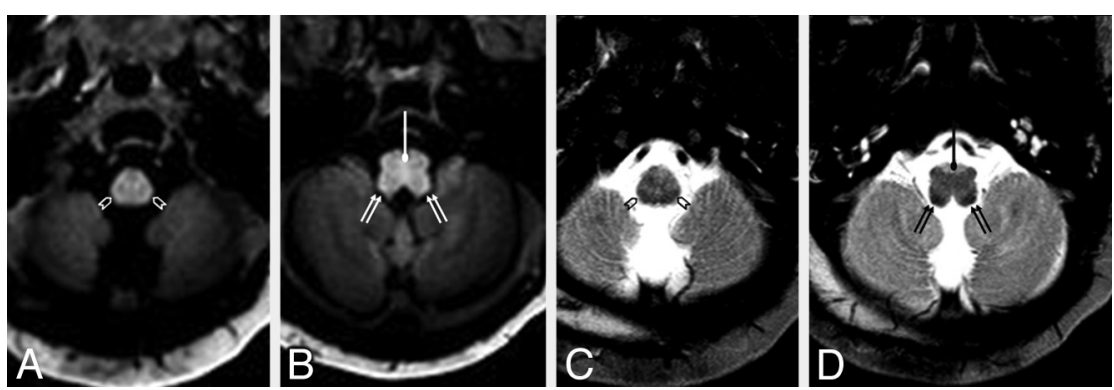

FIG 4. A 4-day-old female neonate with respiratory depression and possible seizures. The pyramidal decussation (circle tipped arrows) is visibly myelinated (bright) on GRE-TTWI ( $A$ and $B$ ) but was rated as "questionably myelinated" (ie, scored \pm by both reviewers on SE-T2WI ( $C$ and $D$ ). In contrast, the spinal tract of V (open arrowheads) was considered to be "definitely myelinated" (ie, scored + ) by both reviewers on SE-T2WI, while it was considered to be only "questionably myelinated" on GRE-TIWI. The ICP (double arrows) appeared clearly myelinated on both sequences. On SE-TIWI (not shown), myelination was not visible (ie, scored as -) by either reviewer in the aforementioned structures in this patient. The ICP was the 1 structure that was clearly myelinated in all neonates on SE-T2WI and was better visualized as being "definitely myelinated" (ie, scored +) on SE-T2WI compared with both GRE-TIWI and SE-TIWI.

cooled $(n=9)$ and noncooled $(n=7)$ patients for each of the 3 sequences. Statistical significance was set to $P<.05$ for each of the above-mentioned calculations.

\section{RESULTS}

Overall, with regard to all structures in all 16 patients, the number of regions counted as "definitely myelinated" $(+)$ was a mean of $64.8 \%$ on GRE-T1WI (range, $57.2 \%-72.4 \%$ by observer), $61.5 \%$ on SE-T2WI (range, 58.2\%-64.8\%), and $36.7 \%$ on SE-T1WI (range, $25.0 \%-$ $48.4 \%)$. The percentages of each structure counted as "+" for myelination based on each sequence are shown in the Table. Both observers found the following structures to be "definitely myelinated" in $\geq 88 \%$ of all patients on GRET1WI: the BIC, DSCP, habenular commissure, medial lemniscus, pyramidal decussation, PLIC, and SCP. In addition, both reviewers noted that the optic tracts were definitely + for myelination in $>80 \%$ of patients on GRE-T1WI. On SE-T2WI, myelination was definitely + in $\geq 88 \%$ of patients within the following structures: BIC, DSCP, ICP, medial lemniscus, and PLIC; both reviewers noted that on SET2WI, the SCP appeared dark (definitely + ) in $\geq 75 \%$ of patients. Regarding SE-T1WI, the only structure that both observers deemed definitely + for myelination in $>88 \%$ of patients was the habenular commissure. Note that 3 structures had a low rate of being + for myelination on all 3 sequences $(\mathrm{a}<40 \%$ myelination rate on each sequence): the callosal splenium, the CST within the brain stem, and the medial longitudinal fasciculus. Examples of myelinated structures on the 3 sequences used are provided in Figs 1-4.

The reviewers agreed overall on $64.0 \%$ of all scores. The interobserver $\kappa$ was moderate-strong for GRE-T1WI $(\kappa=0.546, P<.0001)$, moderate for SE-T2WI $(\kappa=0.468, P<.0001)$, and fair-moderate for SE-T1WI $(\kappa=0.384$, $P<.0001)$. The structures with the highest rate of disagreement between observers were the ICP on GRE-T1WI and SE-T1WI, the PLIC on SE-T1WI, and the pyramidal decussation on SET2WI (Table).

Nine patients had undergone head cooling within the first 72 hours of life; 7 had not. A Mann-Whitney $U$ test showed that overall, there were no significant differences (each $P>.05$ ) among all scores of the cooled-versus-noncooled patients for either reviewer, based on GRE-T1WI $(P=.124-.923)$, SE-T1WI $(P=.794-.884)$, or SE-T2WI $(P=.316-.837)$.

\section{DISCUSSION}

Because the process of myelination is dynamic in the neonate and infant, the determination of the degree of myelination is perhaps 


\begin{tabular}{|c|c|c|c|c|c|c|}
\hline \multirow[b]{2}{*}{ Structure } & \multicolumn{2}{|c|}{ GRE-TIWI } & \multicolumn{2}{|c|}{ SE-TIWI } & \multicolumn{2}{|c|}{ SE-T2WI } \\
\hline & Observer 1 & Observer 2 & Observer 1 & Observer 2 & Observer 1 & Observer 2 \\
\hline $\mathrm{BIC}$ & $100 \%,{ }^{a} 16 / 16$ & $94 \%,{ }^{a} 15 / 16$ & $75 \%, 12 / 16$ & $50 \%, 8 / 16$ & $100 \%,{ }^{a} 16 / 16$ & $88 \%,{ }^{a} 14 / 16$ \\
\hline CCS & $0 \%, 0 / 16$ & $0 \%, 0 / 16$ & $0 \%, 0 / 16$ & $0 \%, 0 / 16$ & $6 \%, 1 / 16$ & $38 \%, 6 / 16$ \\
\hline CNV & $94 \%,{ }^{a} 15 / 16$ & $56 \%, 9 / 16$ & $50 \%, 8 / 16$ & $6 \%, 1 / 16$ & $44 \%, 7 / 16$ & $13 \%, 2 / 16$ \\
\hline CST-BS & $25 \%, 4 / 16$ & $0 \%, 0 / 16$ & $0 \%, 0 / 16$ & $0 \%, 0 / 16$ & $0 \%, 0 / 16$ & $0 \%, 0 / 16$ \\
\hline CST-CS & $75 \%, 12 / 16$ & $63 \%, 10 / 16$ & $75 \%, 12 / 16$ & $31 \%, 5 / 16$ & $6 \%, 1 / 16$ & $6 \%, 1 / 16$ \\
\hline DSCP & $100 \%,{ }^{a} 16 / 16$ & $100 \%,{ }^{a} 16 / 16$ & $81 \%, 13 / 16$ & $31 \%, 5 / 16$ & $100 \%,{ }^{a} 16 / 16$ & $94 \%,{ }^{a} 15 / 16$ \\
\hline $\mathrm{HC}$ & $100 \%,{ }^{a} 16 / 16$ & $100 \%,{ }^{a} 16 / 16$ & $100 \%,{ }^{a} 16 / 16$ & $94 \%,{ }^{a} 15 / 16$ & $69 \%, 11 / 16$ & $63 \%, 10 / 16$ \\
\hline$I C P$ & $88 \%,{ }^{a} 14 / 16$ & $31 \%, 5 / 16$ & $56 \%, 9 / 16$ & $0 \%, 0 / 16$ & $100 \%,{ }^{a} 16 / 16$ & $100 \%,{ }^{a} 16 / 16$ \\
\hline LGN & $38 \%, 6 / 16$ & $25 \%, 4 / 16$ & $6 \%, 1 / 16$ & $0 \%, 0 / 16$ & $50 \%, 8 / 16$ & $56 \%, 9 / 16$ \\
\hline LL & $94 \%,{ }^{a} 15 / 16$ & $69 \%, 11 / 16$ & $38 \%, 6 / 16$ & $56 \%, 9 / 16$ & $75 \%, 12 / 16$ & $44 \%, 7 / 16$ \\
\hline$M L$ & $100 \%,{ }^{a} 16 / 16$ & $94 \%,{ }^{a} 15 / 16$ & $88 \%,{ }^{a} 14 / 16$ & $25 \%, 4 / 16$ & $100 \%,{ }^{a} 16 / 16$ & $100 \%,{ }^{a} 16 / 16$ \\
\hline MLF & $25 \%, 4 / 16$ & $0 \%, 0 / 16$ & $0 \%, 0 / 16$ & $0 \%, 0 / 16$ & $19 \%, 3 / 16$ & $31 \%, 5 / 16$ \\
\hline ON & $44 \%, 7 / 16$ & $44 \%, 7 / 16$ & $0 \%, 0 / 16$ & $0 \%, 0 / 16$ & $19 \%, 3 / 16$ & $44 \%, 7 / 16$ \\
\hline OT & $88 \%,{ }^{a} 14 / 16$ & $81 \%, 13 / 16$ & $69 \%, 11 / 16$ & $56 \%, 9 / 16$ & $56 \%, 9 / 16$ & $56 \%, 9 / 16$ \\
\hline PD & $94 \%,{ }^{a} 15 / 16$ & $88 \%,{ }^{a} 14 / 16$ & $31 \%, 5 / 16$ & $6 \%, 1 / 16$ & $88 \%,{ }^{a} 14 / 16$ & $38 \%, 6 / 16$ \\
\hline PLIC & $100 \%$, $16 / 16$ & $100 \%,{ }^{\mathrm{a}} 16 / 16$ & $100 \%$, $16 / 16$ & $44 \%, 7 / 16$ & $88 \%,{ }^{a} 14 / 16$ & $88 \%,{ }^{a} 14 / 16$ \\
\hline PRC & $56 \%, 9 / 16$ & $19 \%, 3 / 16$ & $31 \%, 5 / 16$ & $6 \%, 1 / 16$ & $88 \%,{ }^{a} 14 / 16$ & $63 \%, 10 / 16$ \\
\hline SCP & $100 \%,{ }^{a} 16 / 16$ & $100 \%,{ }^{a} 16 / 16$ & $75 \%, 12 / 16$ & $44 \%, 7 / 16$ & $88 \%,{ }^{a} 14 / 16$ & $75 \%, 12 / 16$ \\
\hline STV & $56 \%, 9 / 16$ & $25 \%, 4 / 16$ & $44 \%, 7 / 16$ & $25 \%, 4 / 16$ & $81 \%, 13 / 16$ & $69 \%, 11 / 16$ \\
\hline
\end{tabular}

Note:-CCS indicates callosal splenium; CNV, cranial nerve V fascicle; CST-BS, CST in the brain stem; CST-CS, CST in the centrum semiovale; HC, habenular commissure; LGN, lateral geniculate nucleus; LL, lateral lemniscus; ML, medial lemniscus; MLF, medial longitudinal fasciculus; ON, optic nerve; OT, optic tract; PD, pyramidal decussation; PRC, perirolandic cortex; STV, spinal tract of V.

${ }^{\text {a }}$ Structures with $\geq 88 \%$ of patients with myelination.

the most important indicator of brain maturation on MR imaging. ${ }^{3}$ The current study confirms that GRE-T1WI seems to "lead" SE-T1WI in regard to the degree of myelination and has a higher interobserver reliability in subsequently healthy term neonates; this finding is in accordance with a prior 3T study that used the same sequences in subsequently healthy very preterm neonates imaged at term-equivalent age. ${ }^{6}$ However, the current study found slightly higher rates of myelination changes on GRE-T1WI than on SE-T2WI, while the prior analysis of very preterm neonates suggested that SE-T2WI detected myelination as well as or better than GRE-T1WI. ${ }^{6}$ The current results confirm that at $3 \mathrm{~T}$, GRE-T1WI and SE-T2WI should be preferred to SE-T1WI in the assessment of term-neonatal myelination maturation.

While prior studies at lower field strengths have shown that myelination is usually visible on SE-T1WI before SE-T2WI, the findings of this study of myelination on GRE-T1WI and SET2WI, compared with SE-T1WI, could be due to several factors, including field strength. ${ }^{8,9}$ First, compared with the adult brain, the healthy neonatal brain generally demonstrates $\mathrm{T} 1$ prolongation, thought due to a lack of myelin-based lipids such as galactocerebrosides and cholesterol, along with increased water content. ${ }^{8,10-12}$ The high concentration of free water at birth also contributes to the prolonged T2 signal seen in both term and preterm neonate brains at lower field strengths; the absence of certain hydrocarbon elements, such as those found in adult myelinated tissue, may also contribute to such T2 prolongation in early infancy, which could be more pronounced at $3 \mathrm{~T}^{8,12-16}$

As regions mature, the decrease in free water could be less apparent on SE-T1WI at 3T because the bright, T1-shortened signal from maturation is offset by a decrease in spin attenuation. ${ }^{8}$ Also, at 3T, conventional spin-echo sequences are often replaced by an FSE acquisition to decrease scanning time, while attempting to preserve the precision of the myelination evaluation; thus, it is plausible that the increased visibility of dark signal on SE-T2WI at
3T may partially relate to the increasing magnetization transfer effects that occur with an FSE acquisition. ${ }^{17,18}$ Additionally, a GRE-T1WI acquisition at 3T may offer other advantages over SE-T1WI; for example, GRE-T1WI has shown a higher SNR gain compared with FSE T1WI techniques at $3 \mathrm{~T}$ in adult brains. ${ }^{19}$ Hence, such known effects may account for the greater visibility of myelination on GRE-T1WI and SE-T2WI at 3T in neonates, compared with SE-T1WI, based on the results of this study and the prior one. ${ }^{6}$ However, such theoretic benefits in evaluating myelination at higher field strengths are speculative, without direct comparison among field strengths or corroboration by histology.

The noted differences in the frequency of maturation of structures among sequences, as exemplified in the current study by the habenular commissure, ICP, and perirolandic cortex, could be explained by some of the complex interactions mentioned above, including the use of FSE acquisitions, the concentration of free water surrounding a structure, and other factors related to the composition of myelin or its precursors. ${ }^{18,20-23}$ Unfortunately, many MR imaging studies of myelination in neonates do not consistently evaluate the same set of structures, particularly the habenular commissure, for example. It has been noted previously that there can be substantial discrepancies both among the literature and among sequences regarding myelin maturation; for example, 1 noted factor has been that magnetization transfer effects may augment the visualization of maturation on FSE-T2WI versus conventional SE-T2WI. ${ }^{18,21,22}$ While conventional knowledge is that T1WI is more useful for early myelination changes and T2WI is better for later myelination changes, there are studies that suggest that certain structures may preferentially manifest the initial changes of maturation on T2WI; also, Barkovich ${ }^{2}$ has suggested that myelination of gray matter structures may be best demonstrated on T2WI, whereas white matter structures may be better visualized on T1WI. ${ }^{2,14,23}$ Given such variations in myelination among sequences and among the literature at lower field 
strengths, it is conceivable that such differences would theoretically be exacerbated at higher fields strengths such as 3T.

An important finding in the current study was that the apparent discrepancy regarding the frequency of T1-bright myelination of the CST within the brain stem was less than that of the PLIC, though maturation is known to occur earlier within the CST within the brain stem both histologically and at lower field strengths (eg, 0.6T, 1T). ${ }^{14,24}$ This appearance could be due to a complex interaction of various factors in the current $3 \mathrm{~T}$ study: the composition of myelin (or its precursors) in that location, the presence of free water, the "compactness" of fibers, or the field strength. As stated above, the T1-bright, T2-dark appearance of the dynamic process of myelination likely relates to a complex interaction of the progressively decreasing free water content along with cholesterol, galactocerebrosides, and proteins of the myelin membrane..$^{8,10,11,20,21}$ It is conceivable that a site with a higher degree of "compactness" of fibers, as evidenced by the described higher fractional anisotropy in the PLIC relative to the CST within the brain stem, could have brighter T1 signal due to a higher concentration of myelin or myelin precursors. ${ }^{25,26}$ Such lower "compactness" of fibers in the CST within the brain stem, albeit being myelinated earlier, in tandem with the known surrounding higher free water content of the ventral pons in general, could theoretically obscure what should appear as T1-bright signal intensity. This appearance may be even more profound at higher field strengths such as 3T, due to its increasing ability to depict free water. ${ }^{14,20,24}$ Thus, this discrepancy between the current study and histology is likely an MR imaging discrepancy at 3T that radiologists and clinicians should be aware of. Therefore, because the CST within the brain stem is known to be myelinated earlier than the PLIC, the lack of T1-bright signal within the CST within the brain stem on 3T MR imaging should not be misconstrued as a lack of maturation in this location.

Another interesting finding of this study was that on all sequences, the callosal splenium exhibited a much lower rate of myelination $(<40 \%)$ on SE-T2WI compared with the rate in the prior study of very preterm neonates at term-equivalent age (>90\%). ${ }^{6}$ The difference between these cohorts deserves some discussion as to possible factors. One is that, while the growth of the callosal genu slows after birth, in contrast, splenial growth accelerates after birth; perhaps this finding could represent accelerated myelination in the cohort of very preterm neonates. ${ }^{27}$ However, diffusion tensor imaging studies that have focused on the callosal splenium have shown disparate results; 1 study of preterm infants at term-equivalent age found significant differences compared with term infants, while another found no definite relationship between gestational age and changes in various DTI parameters. ${ }^{28,29}$ Theoretically, another possibility may relate to a phenomenon called the "visual experience" hypothesis, which essentially states that because preterm infants are "out" of the mother longer by the time of term-equivalent imaging (relative to full-term neonates), healthy premature infants would have experienced a greater degree of visual stimulation and thus would have accelerated maturation of visual pathways by the same postnatal gestational age. ${ }^{30}$ Most interesting, studies using visualevoked potentials and contrast sensitivities have suggested accelerated maturation of the visual cortices in preterm relative to term infants, which might have manifested in the current study as earlier visualization of callosal splenium maturation in the preterm infants imaged at term-equivalent age. ${ }^{30,31}$ However, this finding on MR imaging is difficult to prove without correlating it with other clinical tests.

Regarding the interobserver reliability, a potential criticism of this study would be that the interobserver reliability was not high, because GRE-T1WI was moderate-strong $(\kappa=0.546)$, SE-T2WI was moderate $(\kappa=0.468)$, and SE-T1WI was fair-moderate ( $\kappa=$ $0.384)$. We opine that the findings of moderate-strong interobserver reliability highlight how the task of assessing myelination in young infants can be challenging, which has been mentioned previously. ${ }^{21}$ Such variability is quite similar to that in the prior study of very preterm neonates at term-equivalent age, in which GRET1WI was moderate-high $(\kappa=0.56)$ and both SE-T2WI $(\kappa=$ $0.43)$ and SE-T1WI $(\kappa=0.40)$ were moderate. ${ }^{6}$ Our review of the limited available literature on the subject of interobserver reliability in term neonates found only a few reports even at lower field strengths, with the interobserver $\kappa$ ranging from 0.21 to 0.40 by using routine sequences in early infancy. ${ }^{3,6,22,32}$ One study of solely very preterm neonates found a much higher interobserver reliability $(\kappa=0.72)$ when using SE-T1WI, SE-T2WI, and inversion recovery T1WI at $1 \mathrm{~T}$, but that study analyzed fewer structures and did not provide the $\kappa$ for each sequence; thus, their results are difficult to extrapolate onto the current study. ${ }^{3}$ It is also plausible that there is lower interobserver reliability at $3 \mathrm{~T}$ compared with lower field strengths, but this aspect was not explored by the current study. Hence, we opine that such interobserver variability is likely present even among experienced pediatric neuroradiologists who routinely assess myelination, due to subtle changes in early infancy, but this phenomenon is perhaps underreported. Therefore, we think that such variability underscores the need to use GRE-T1WI at a minimum when assessing myelination at $3 \mathrm{~T}$.

The limitations of this study include those inherently involved with retrospective analyses. Also, a qualitative analysis based on subjective scoring was used, in the fashion of the prior report of preterm neonates. ${ }^{6}$ Additionally, the number of participants was limited, because MR imaging was only performed when there was clinical concern for hypoxic-ischemic injury; this limitation could have caused selection bias because 9 of the 16 patients had undergone head-cooling therapy before the MR imaging. Thus, it is plausible that a difference in myelination may exist between cooled and noncooled patients; however, we found no significant difference between those 2 groups regarding the degree of myelinated structures. Finally, another potential limitation is that a minimum clinical follow-up of 6 months may not have been enough to entirely ensure a lack of mild neurologic sequelae.

\section{CONCLUSIONS}

GRE-T1WI depicts a higher degree of myelination than SE-T1WI and has a higher interobserver reliability than both SE-T2WI and SE-T1WI. Compared with a prior 3T study of very preterm infants at term-equivalent age, in which SE-T2WI showed a slightly higher overall rate of myelination in very preterm infants, our study found that GRE-T1WI had a slightly higher rate of myelination than SE-T2WI. These findings confirm that in both term 
neonates and term-equivalent age infants, myelination changes are best evaluated on GRE-T1WI and SE-T2WI at 3T, while the performance of SE-T1WI at $3 \mathrm{~T}$ is inferior in assessing the degree of neonatal myelination.

\section{REFERENCES}

1. Sie LT, van der Knaap MS, van Wezel-Meijler G, et al. MRI assessment of myelination of motor and sensory pathways in the brain of preterm and term-born infants. Neuropediatrics 1997;28:97-105

2. Barkovich AJ. MR of the normal neonatal brain: assessment of deep structures. AJNR Am J Neuroradiol 1998;19:1397-403

3. Counsell SJ, Maalouf EF, Fletcher AM, et al. MR imaging assessment of myelination in the very preterm brain. AJNR Am J Neuroradiol 2002;23:872-81

4. Lu H, Nagae-Poetscher LM, Golay X, et al. Routine clinical brain MRI sequences for use at 3.0 Tesla. J Magn Reson Imaging 2005; 22:13-22

5. Schmitz BL, Grön G, Brausewetter F, et al. Enhancing gray-to-white matter contrast in 3T T1 spin-echo brain scans by optimizing flip angle. AJNR Am J Neuroradiol 2005;26:2000-04

6. Sarikaya B, McKinney AM, Spilseth B, et al. Comparison of spinecho T1- and T2-weighted and gradient-echo T1-weighted images at $3 \mathrm{~T}$ in evaluating very preterm neonates at term-equivalent age. AJNR Am J Neuroradiol 2013;34:1098-103

7. Naidich TP, Duvernoy HM, Delman BN, et al. Duvernoy's Atlas of the Human Brain Stem and Cerebellum. New York: Springer-Verlag; 2009

8. Barkovich AJ, Kjos BO, Jackson DEJ, et al. Normal maturation of the neonatal and infant brain: MR imaging at 1.5 T. Radiology 1988; $166: 173-80$

9. Martin E, Kikinis R, Zuerrer M, et al. Developmental stages of human brain: an MR study. J Comput Assist Tomogr 1988;12:917-22

10. Koenig SH, Brown RD 3rd, Spiller M, et al. Relaxometry of brain: why white matter appears bright in MRI. Magn Reson Med 1990; 14:482-95

11. Kucharczyk W, Macdonald PM, Stanisz GJ, et al. Relaxivity and magnetization transfer of white matter lipids at MR imaging: importance of cerebrosides and pH. Radiology 1994;192:521-29

12. Dobbing J, Sands J. Quantitative growth and development of human brain. Arch Dis Child 1973;48:757-67

13. Thornton JS, Amess PN, Penrice J, et al. Cerebral tissue water spinspin relaxation times in human neonates at 2.4 Tesla: methodology and the effects of maturation. Magn Reson Imaging 1999;17:1289-95

14. Counsell SJ, Kennea NL, Herlihy AH, et al. T2 relaxation values in the developing preterm brain. AJNR Am J Neuroradiol 2003;24: $1654-60$

15. Williams LA, Gelman N, Picot PA, et al. Neonatal brain: regional variability of in vivo $M R$ imaging relaxation rates at $3.0 \mathrm{~T}$ : initial experience. Radiology 2005;235:595-603
16. Holland BA, Haas DK, Norman D, et al. MRI of normal brain maturation. AJNR Am J Neuroradiol 1986;7:201-08

17. Prenger EC, Beckett WW, Kollias SS, et al. Comparison of T2weighted spin-echo and fast spin-echo techniques in the evaluation of myelination. J Magn Reson Imaging 1994;4:179-84

18. Shaw DW, Weinberger E, Astley SJ, et al. Quantitative comparison of conventional spin echo and fast spin echo during brain myelination. J Comput Assist Tomogr 1997;21:867-71

19. Wintersperger BJ, Runge VM, Biswas J, et al. Brain tumor enhancement in MR imaging at 3 Tesla: comparison of SNR and CNR gain using TSE and GRE techniques. Invest Radiol 2007;42:558-63

20. Barkovich AJ. Concepts of myelin and myelination in neuroradiology. AJNR Am J Neuroradiol 2000;21:1099-109

21. Welker KM, Patton A. Assessment of normal myelination with magnetic resonance imaging. Semin Neurol 2012;32:15-28

22. Kizildağ B, Düşünceli E, Fitoz $S$, et al. The role of classic spin echo and FLAIR sequences for the evaluation of myelination in MR imaging. Diagn Interv Radiol 2005;11:130-36

23. Murakami JW, Weinberger E, Shaw DW. Normal myelination of the pediatric brain imaged with fluid-attenuated inversion-recovery (FLAIR) MR imaging. AJNR Am J Neuroradiol 1999;20:1406-11

24. McArdle CB, Richardson CJ, Nicholas DA, et al. Developmental features of the neonatal brain: MR imaging. Part I. Gray-white matter differentiation and myelination. Radiology 1987;162:223-29

25. Partridge SC, Mukherjee P, Berman JI, et al. Tractography-based quantitation of diffusion tensor imaging parameters in white matter tracts of preterm newborns. J Magn Reson Imaging 2005; 22:467-74

26. Partridge SC, Vigneron DB, Charlton NN, et al. Pyramidal tract maturation after brain injury in newborns with heart disease. Ann Neurol 2006;59:640-51

27. Kier EL, Truwit CL. The normal and abnormal genu of the corpus callosum: an evolutionary, embryologic, anatomic, and MR analysis. AJNR Am J Neuroradiol 1996;17:1631-41

28. Hasegawa T, Yamada K, Morimoto M, et al. Development of corpus callosum in preterm infants is affected by the prematurity: in vivo assessment of diffusion tensor imaging at term-equivalent age. $\mathrm{Pe}$ diatr Res 2011;69:249-54

29. De Bruïne FT, van Wezel-Meijler G, Leijser LM, et al. Tractography of developing white matter of the internal capsule and corpus callosum in very preterm infants. Eur Radiol 2011;21:538-47

30. Bosworth RG, Dobkins KR. Effects of prematurity on the development of contrast sensitivity: testing the visual experience hypothesis. Vision Res 2013;82:31-41

31. Leaf AA, Green CR, Esack A, et al. Maturation of electroretinograms and visual evoked potentials in preterm infants. Dev Med Child Neurol 1995;37:814-26

32. Maricich SM, Azizi P, Jones JY, et al. Myelination as assessed by conventional MR imaging is normal in young children with idiopathic developmental delay. AJNR Am J Neuroradiol 2007;28: 1602-05 